\title{
Transverse flow of charmed hadrons in relativistic heavy-ion collisions
}

\author{
P. Girigori and T. Peitzmann \\ Universiteit Utrecht/NIKHEF, NL-3508 TA Utrecht, The Netherlands
}

(Received 28 April 2009; published 19 August 2009)

\begin{abstract}
Transverse mass spectra for D mesons are calculated in 1+1-dimensional hydrodynamics using different equations of state. Parameters of the simulations were tuned to describe proton spectra in heavy-ion collisions at RHIC. The assumption that the initial distribution of charm follows a scaling of binary collisions different from the bulk matter leads to significantly smaller effective slopes and mean $p_{T}$ value of the D-meson spectra, similar to the effect of a possible higher freeze-out temperature. This has to be taken into account in the interpretation of such spectra in the context of hydrodynamical models.
\end{abstract}

DOI: 10.1103/PhysRevC.80.027901

PACS number(s): 25.75.Ld

Recent data from relativistic heavy-ion collisions at RHIC have demonstrated very interesting properties of the matter produced [1-4]. In particular, the medium shows very strong elliptic flow, requiring collectivity, and appears to be opaque for hard scattered partons, which calls for very high initial densities. Although the behavior of light and strange hadrons seems to fit consistently into an emerging picture of heavy-ion reactions, the description of charmed (or heavier) hadrons is one of the remaining puzzles. Single electrons, presumably originating from charm and bottom decays, show a suppression in central $\mathrm{Au}+\mathrm{Au}$ collisions very similar to that of light hadrons [5,6], which is not easily explained by models of parton energy loss [7]. In addition these electrons show significant elliptic flow, calling for a strong coupling of the heavy hadrons to the collective motion of the medium [8].

In a recent study charm production in $\mathrm{Au}+\mathrm{Au}$ collisions at RHIC is compared to hydrodynamic parametrizations [9]. The so-called blast-wave model of the momentum spectra with parameters consistent with light and/or strange hadron spectra does not describe the spectra of single electrons. As the measurement of elliptic flow seems to indicate early equilibration, this failure would imply that heavy hadrons should decouple from the bulk matter at a much higher temperature than light hadrons. However, blast-wave models, but also more serious implementations of hydrodynamics, still suffer from certain approximations, which may be relevant for the interpretation of the effects of collective flow seen in heavy hadrons.

One such approximation is the assumption that all particle species share the same initial density distribution. However, whereas the amount of bulk matter, dominantly converted into pions in the final state, should scale more like the number of participating nucleons [10], the production of heavy hadrons is expected to scale with the number of binary nucleon-nucleon collisions, as for any hard process. These different production probabilities should result in different initial transverse distributions for heavy compared to light hadrons. Even for complete equilibration, where heavy flavor would be carried with the flow of the bulk, and simultaneous freeze-out, the velocity distribution of the effective sources of the final state particles would be different and would possibly modify the momentum spectra of heavy hadrons. In this Brief Report, we will study the effect of a different initial density distribution for a rare particle species on its final spectrum in a hydrodynamic calculation.

Our simulations are based on the Monte Carlo program by Romatschke for 1+1-dimensional hydrodynamics [11,12]. This model uses Bjorken scaling and includes effects of viscosity. It was initially drafted with an ideal equation of state (IdEOS) to close the set of hydrodynamical equations. To analyze the effects of the chosen (EOS) on the hydrodynamical evolution of the system we have added an EOS with a transition from the quark-gluon plasma (QGP) phase to a hadronic resonance gas $(\mathrm{PhEOS})$ parametrized according to

$$
p= \begin{cases}\frac{1}{3}\left(\epsilon-4 \epsilon_{0}\right)+c_{0} & \text { if } \epsilon>\epsilon_{\mathrm{QGP}}, \\ 0.02 \epsilon+c_{1} & \text { if } \epsilon_{\mathrm{HG}} \leqslant \epsilon \leqslant \epsilon_{\mathrm{QGP}}, \\ \frac{1}{5} \epsilon & \text { if } \epsilon<\epsilon_{\mathrm{HG}},\end{cases}
$$

where

$$
\begin{aligned}
\epsilon_{\mathrm{QGP}}\left(T_{c}\right) & =\frac{5}{3}\left[\epsilon\left(T_{c}\right)-4 \epsilon_{0}\right], \\
\epsilon_{\mathrm{HG}}\left(T_{c}\right) & =\epsilon\left(T_{c}\right) .
\end{aligned}
$$

This corresponds to a critical temperature $T_{c}=164 \mathrm{MeV}$ and a latent heat of $\epsilon_{\mathrm{QGP}}-\epsilon_{\mathrm{HG}} \sim 1.35 \mathrm{GeV} / \mathrm{fm}^{3}$. The parameters $c_{0}$ and $c_{1}$ are determined by requiring continuity of $p(\epsilon)$. Figure 1 shows both equations of state employed during this analysis. ${ }^{1}$ If not mentioned otherwise, we have employed a negligible viscosity of $\eta / s=10^{-4}$. Calculations have also been performed for $\eta / s=0.08$.

The initial transverse density distribution for the bulk matter, which determines, for example, the yield of light flavors, is derived from the participant density. We have also added a conserved quantum number to the simulation, which can be used to describe heavy flavor. We will refer to it as charm in the following. The absolute normalization of this charm contribution is arbitrary as we are only interested in spectral shapes. The charm can be distributed at $t=0$ according to an arbitrary distribution - in addition to the participant density (WN) we will use a distribution following the density of binary

\footnotetext{
${ }^{1}$ An EOS based on lattice QCD would be most appropriate. However, as our main purpose of using different equations of states is to demonstrate the robustness of the results, we have for simplicity used these choices.
} 


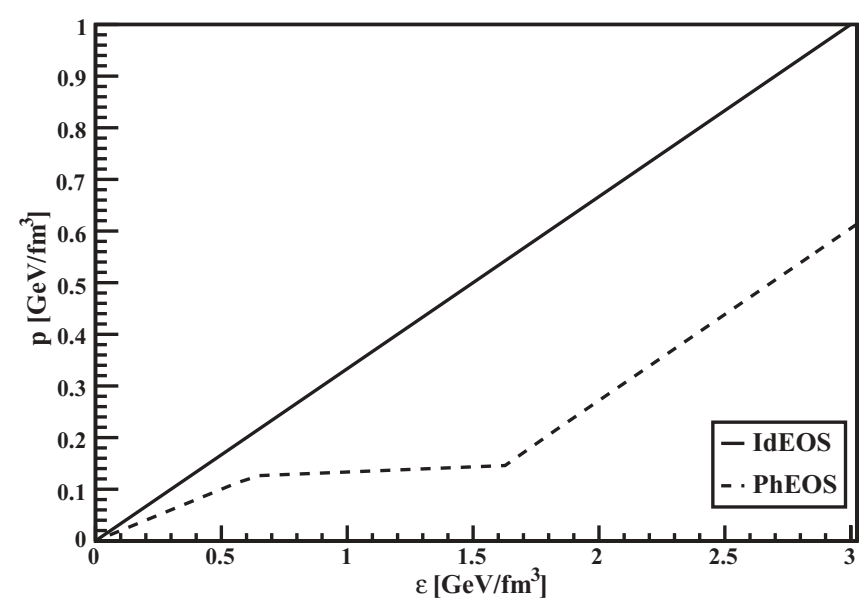

FIG. 1. Pressure as a function of energy density for the two equations of state as employed in the present calculation.

collisions (BC). During the evolution the charm will flow along with the bulk matter.

The evolution for an initial density following binary collisions can be seen from Fig. 2, which shows the relative number densities in the transverse direction of this distribution for the charm (C) to light (L) flavor at different times during the evolution. This hydrodynamical evolution was performed using IdEOS. The charm is distributed differently from the light flavor, which follows the participant density. The distribution of the binary collision density is more strongly peaked in the center and hence leads to a larger concentration of charm to light in the inner regions. As the system evolves and velocity builds up, the relative number densities increase in the outer regions, but at decoupling the difference will still be significant. A similar evolution is observed for the PhEOS.

We calibrated the model by comparing the proton spectra generated from the hydrodynamical model to experimental data taken by the PHENIX experiment for the 5\% most central $\mathrm{Au}+\mathrm{Au}$ collisions [13], where a feed-down correction for $\Lambda$ decays has been applied. The transverse mass distribution

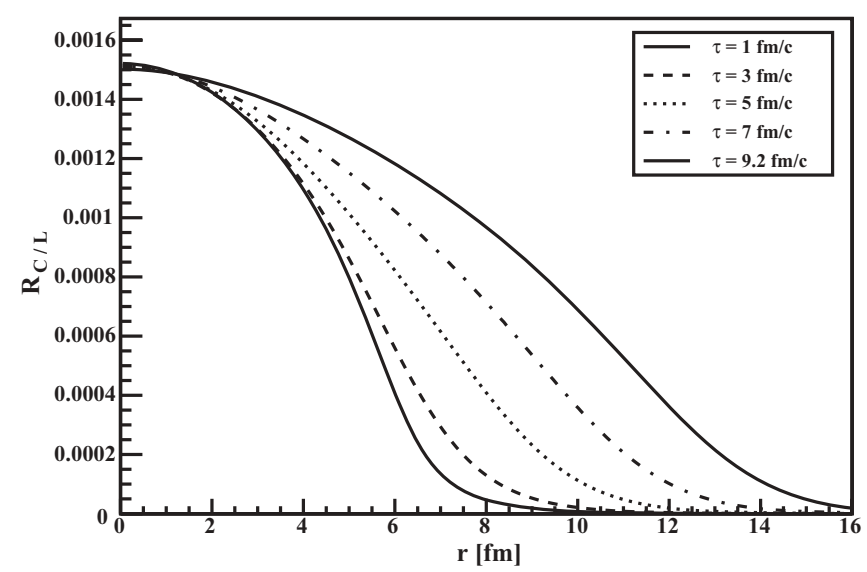

FIG. 2. Relative number densities as a function of the radius of light and heavy (charmed) flavor at different times during the hydrodynamic evolution. IdEOS was applied during this simulation for the EOS.

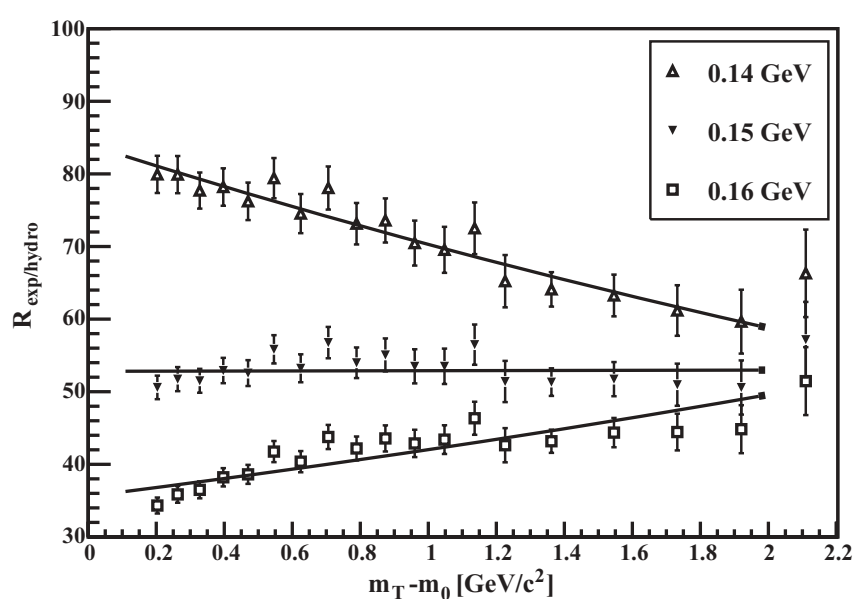

FIG. 3. Ratio of transverse mass distributions of protons in central $\mathrm{Au}+\mathrm{Au}$ collisions measured by PHENIX to the results of the hydrodynamical simulation for IdEOS.

of the proton were computed using the noninstantaneous freeze-out prescription for different initial and freeze-out temperatures. After deciding on a best initial temperature we then proceeded to plot the ratio of the spectra generated from the hydrodynamical model to the spectra obtained in the experiments for different freeze-out temperatures. As significant for this comparison we have used the range $0 \leqslant m_{T}-m_{0} \leqslant 2$ for the proton spectra. The result for IdEOS can be seen in Fig. 3. The best parameters found for the initial and freeze-out temperature were $T_{i}=0.40 \mathrm{GeV}$ and $T_{\mathrm{fo}}=$ $0.15 \mathrm{GeV}$, respectively. The calculations for these parameter values also yield reasonable descriptions of the pion spectra as measured in PHENIX and the proton and pion spectra for the $12 \%$ most central $\mathrm{Au}+\mathrm{Au}$ collisions from STAR.

The model was also calibrated as explained here for the hydrodynamical evolution of the EOS with a phase transition. The best parameters found for the initial and freeze-out temperature were $T_{i}=0.36 \mathrm{GeV}$ and $T_{\mathrm{fo}}=0.14 \mathrm{GeV}$, respectively. Similar parameters were found for the calculations with finite viscosity; for a summary see Table I.

Having calibrated the model we proceeded to compute the transverse mass spectrum of a charmed hadron (i.e., the D meson) using different initial distributions as the effective source of the final state distribution of the particle. The momentum spectra of the charm have been calculated using the Cooper-Frye prescription for noninstantaneous freeze-out,

$$
\begin{aligned}
\frac{d n}{m_{\mathrm{T}} d m_{\mathrm{T}}}= & C m_{\mathrm{T}} \int r d r \tau_{\mathrm{fo}}(r) c(r)\left[\mathrm{K}_{1}\left(\frac{m_{\mathrm{T}} u^{\tau}}{T_{\mathrm{fo}}}\right) I_{0}\left(\frac{p_{T} u^{r}}{T_{\mathrm{fo}}}\right)\right. \\
& \left.-\frac{d \tau_{\mathrm{fo}}}{d r} p_{T} \mathrm{~K}_{0}\left(\frac{m_{T} u^{\tau}}{T_{\mathrm{fo}}}\right) \mathrm{I}_{1}\left(\frac{p_{T} u^{r}}{T_{\mathrm{fo}}}\right)\right]
\end{aligned}
$$

where $d \tau_{\mathrm{fo}} / d r$ was calculated with the finite differences between subsequent freeze-out radii (i.e., $\Delta \tau_{\mathrm{fo}} / \Delta r$ ). Furthermore, $c(r)$ is the number density at a freeze-out radius, $u^{\tau}$ and $u^{r}$ are the velocity components at freeze-out, $T_{\mathrm{fo}}$ is the freeze-out temperature, and $\tau_{\mathrm{fo}}$ is the time at which a certain radius has decoupled. 
TABLE I. Parameters for the hydrodynamical calculations $\left(\eta / s=10^{-4}\right)$ and results for mean $p_{T}$ and effective slopes.

\begin{tabular}{|c|c|c|c|c|c|c|c|}
\hline & \multirow[t]{2}{*}{$T_{i}(\mathrm{GeV})$} & \multirow[t]{2}{*}{$T_{\text {fo }}(\mathrm{GeV})$} & \multirow[t]{2}{*}{$\left\langle p_{T}\right\rangle_{\pi}(\mathrm{GeV} / c)$} & \multicolumn{2}{|c|}{$\left\langle p_{T}\right\rangle_{D}(\mathrm{GeV} / c)$} & \multicolumn{2}{|c|}{$T_{\mathrm{eff}, \mathrm{D}}^{\left(0.7 \mathrm{GeV} / c^{2}\right)}(\mathrm{GeV} / c)$} \\
\hline & & & & WN & $\mathrm{BC}$ & WN & $\mathrm{BC}$ \\
\hline IdEOS & 0.40 & 0.15 & 0.60 & 1.64 & 1.55 & 0.62 & 0.54 \\
\hline PhEOS & 0.36 & 0.14 & 0.58 & 1.69 & 1.61 & 0.68 & 0.58 \\
\hline
\end{tabular}

The spectra for both initial distributions and both equations of state are given in Fig. 4 on the left. The spectra of the $\mathrm{D}$ meson for the different initial distributions were arbitrarily scaled, since only the shape of the spectra is of interest for this analysis. All spectra are similar in shape as expected, so for a detailed comparison it is more instructive to look at ratios. On the right side of Fig. 4 one can see ratios of spectra for $\mathrm{BC}$ scaling to those for $\mathrm{WN}$ scaling for the different equations of state (upper plot) and similarly the ratio for IdEOS to $\mathrm{PhEOS}$ for the different initial distributions (lower plot). One can see that the calculations for the $\mathrm{BC}$ initial distribution predict relatively more yield at small $m_{T}-m_{0}$. Also PhEOS predicts a slightly lower yield for low-momentum D mesons $\left(m_{T}-m_{0} \leqslant 1 \mathrm{GeV} / c^{2}\right)$ compared to IdEOS, as seen in the bottom right panel of Fig. 4 .

To quantify the differences in the spectra we calculated local inverse slope parameters and the overall average $p_{T}$. The first technique is based on the fact that the spectral shape as a function of transverse mass can locally be characterized by an exponential with a particular effective slope. The effective slopes were calculated for bin intervals of $\Delta\left(m_{T}-m_{0}\right)=$ $0.2 \mathrm{GeV} / c^{2}$ and given for IdEOS and PhEOS for both initial distributions in Fig. 5. From these results we can see that different initial distributions do affect the shape of the particle spectra for both equations of state. PhEOS results in higher effective slopes than IdEOS for small momentum. For both equations of state the effective slope for a $\mathrm{BC}$ initial distribution is $\sim 10 \%-20 \%$ smaller than for the $\mathrm{WN}$ distribution for $m_{T}-m_{0} \leqslant 1 \mathrm{GeV} / c^{2}$. At large momentum the differences between the chosen initial distributions decrease up to the point where they cannot be distinguished from each other.
Such an effect is qualitatively expected for a narrower initial distribution. Charm is less likely emitted from source elements at large radii, which will at freeze-out acquire larger velocities. The smaller contribution from high velocities leads to smaller high-momentum components in the spectra.

The results for the calculation of the mean transverse momentum for D mesons are given in Table I together with the mean transverse momentum of pions. The mean $p_{T}$ of pions is high compared to experimental values because resonance decays have not been taken into account here. The different initial distributions result in different values for the mean transverse momentum. Note that the mean transverse momentum for the PhEOS results for the D-meson spectra are larger compared to the IdEOS results, in line with the analysis of the slopes, and again the BC initial distribution yields a significantly smaller mean $p_{T}$ than $\mathrm{WN}$ for both equations of state. There are slight differences in the mean transverse momentum of pions for the two equations of state, which are likely related to the fact that the models have been tuned to the proton spectra.

We have also performed calculations for a finite viscosity $(\eta / s=0.08)$. Figure 6 shows inverse slopes of the resulting spectra for D mesons determined by assuming instantaneous freeze-out. The inverse slopes depend only weakly on viscosity. They are slightly different compared to calculations made by assuming noninstantaneous freeze-out, but the difference between the slopes for the two different initial distributions is similar in magnitude in all cases.

We have studied the effects of the initial distribution for a rare particle species on its spectrum using different equations of state. The simulations are based on Romatschke's
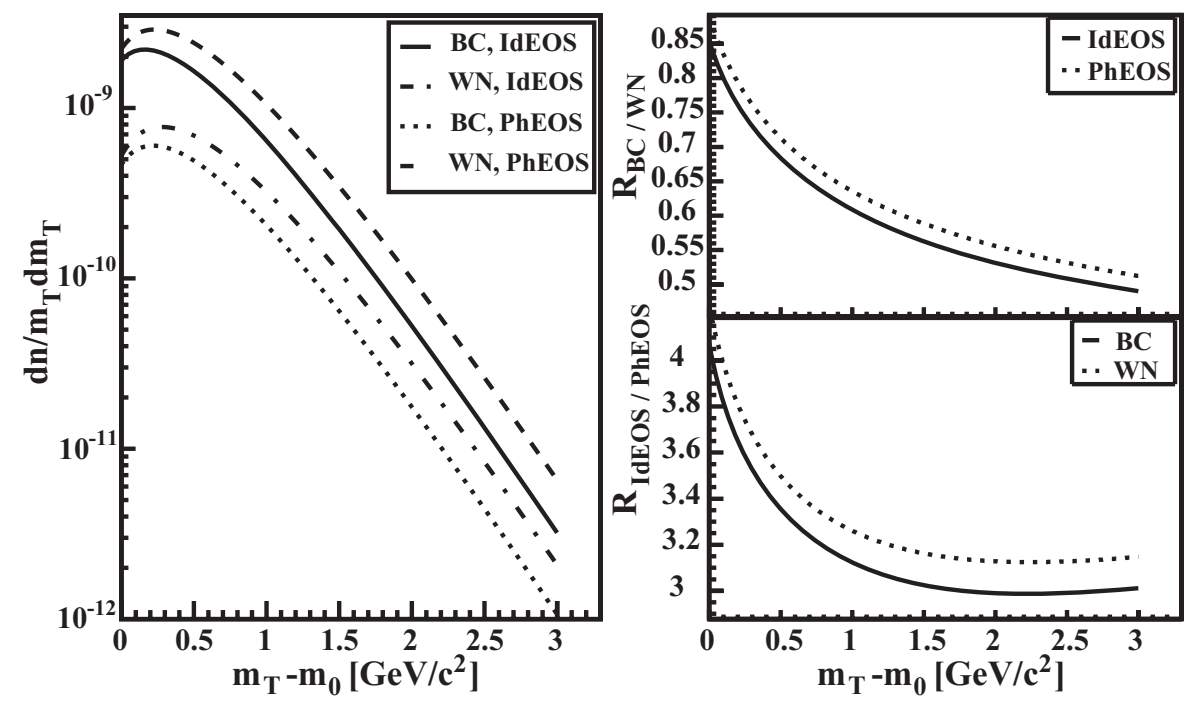

FIG. 4. (Left) Transverse mass distributions of $\mathrm{D}$ mesons from the hydrodynamics simulation for the different equations of state and initial distributions. (Upper right) The ratio of the spectra for binary collision initial distributions to the participant distributions. (Lower right) The ratio of the spectra for the ideal EOS to the EOS with a phase transition. 

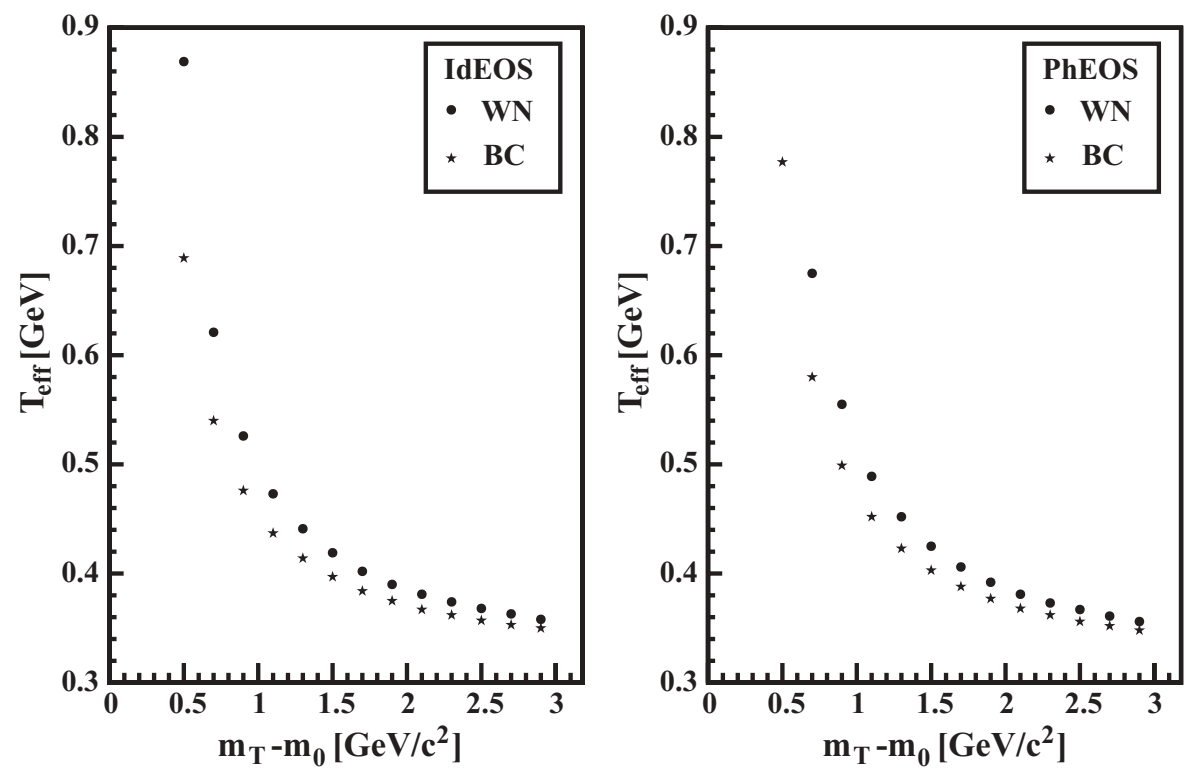

FIG. 5. Effective slope of the transverse mass spectra of $\mathrm{D}$ mesons for the two initial distributions of interest, for the different equations of state as described in the legend. 1+1-dimensional viscous hydrodynamical model. Our model calculations are calibrated by comparing the proton spectrum obtained from the hydrodynamical model to the proton spectrum as measured by the PHENIX experiment in central $\mathrm{Au}+\mathrm{Au}$ collisions. For IdEOS we found as optimal parameters $T_{i}=0.40 \mathrm{GeV}$ and $T_{\mathrm{fo}}=0.15 \mathrm{GeV}$ for the initial and freeze-out temperature, respectively. PhEOS resulted in

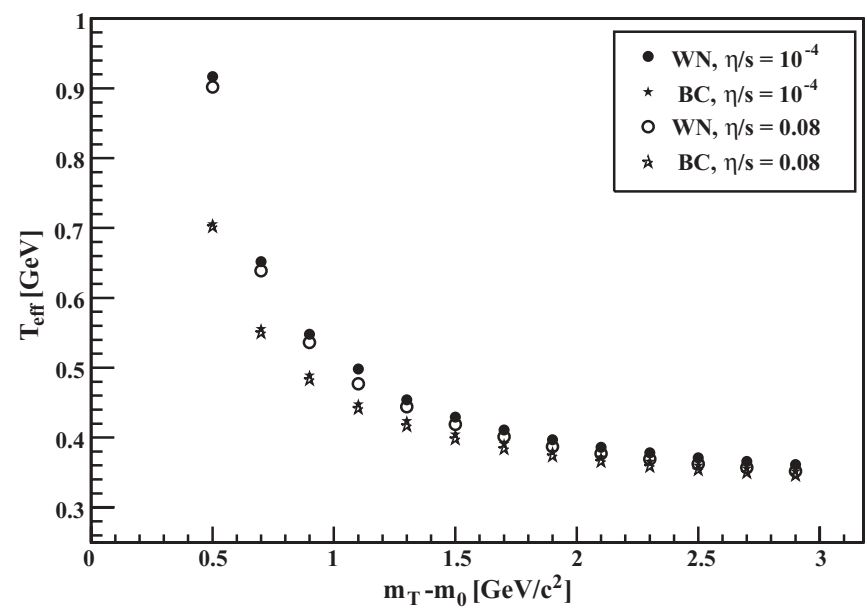

FIG. 6. Effective slope of the transverse mass spectra of D mesons for the two initial distributions as before, for the EOS with phase transition and different viscosities as described in the legend. Spectra have been calculated for instantaneous freeze-out in this case. the best parameters $T_{i}=0.36 \mathrm{GeV}$ and $T_{\mathrm{fo}}=0.14 \mathrm{GeV}$ for the initial and freeze-out temperatures, respectively.

From the transverse distribution of the relative number densities it can be seen that a difference in the initial distribution of charm compared to the light flavors survives the hydrodynamical evolution. This difference was still significant at decoupling. The spectra of the $\mathrm{D}$ meson for two different initial distributions have been calculated and their spectral shape was analyzed for both equations of state by using inverse slope parameters and the mean transverse momentum. Both methods show that the initial distribution does affect the spectral shape and must therefore be taken into account when conducting model studies. An initial distribution following a scaling with binary collisions results in smaller inverse slopes and mean transverse momentum. This conclusion is not affected by small finite viscosities.

This effect is important for the interpretation of charmed particle spectra in the context of hydrodynamical models. The reduction of mean transverse momentum from this mechanism is qualitatively similar to the effect one expects, for example, for the possible freeze-out of charmed hadrons at higher temperatures. Before claims of different freeze-out temperatures from measured spectral shapes can be made, the effect studied here has to be taken into account.

We would like to thank P. Romatschke for providing his computer program and for valuable comments and suggestions.
[1] I. Arsene et al., Nucl. Phys. A757, 1 (2005).

[2] K. Adcox et al., Nucl. Phys. A757, 184 (2005).

[3] B. B. Back et al., Nucl. Phys. A757, 28 (2005).

[4] J. Adams et al., Nucl. Phys. A757, 102 (2005).

[5] S. S. Adler et al., Phys. Rev. Lett. 96, 032301 (2006).

[6] B. I. Abelev et al., Phys. Rev. Lett. 98, 192301 (2007).

[7] I. Vitev, J. Phys. G 35, 104011 (2008).

[8] A. Adare et al., Phys. Rev. Lett. 98, 172301 (2007).
[9] B. I. Abelev et al., arXiv:0805.0364.

[10] G. I. Veres et al., presented at the 20th International Conference on Nucleus-Nucleus Collisions (Quark Matter 2008), Jaipur, Rajasthan, India, 4-10 February 2008, arXiv:0806.2803.

[11] R. Baier and P. Romatschke, Eur. Phys. J. C 51, 677 (2007).

[12] R. Baier, P. Romatschke, and U. A. Wiedemann, Phys. Rev. C 73, 064903 (2006).

[13] S. Adler et al., Phys. Rev. C 69, 034909 (2004). 